\title{
CRIANÇAS NASCIDAS APÓS EMPREGO DE TÉCNICA DE FERTILIZAÇÃO ASSISTIDA
}

\section{CHILDREN BORN AFTER ASSISTED REPRODUCTION TECHNIQUES}

\author{
Marcia de Freitas * \\ Conceição Aparecida de Mattos Segre ** \\ Jonathas Soares Borge **k \\ Sidney Glinav ***⿰㇇⿰亅⿱丿丶丶 \\ Claudio Leone **w*k \\ Arnaldo Augusto Franco de Siqueira *⿻丷木*⿲丶丶㇒木火
}

Freitas M, et al. Crianças Nascidas após Emprego de Técnica de Fertilização Assistida. Rev Bras Crescimento Desenvolvimento Hum. 2008; 18(3): 218-228

\section{Resumo:}

Objetivo: Caracterizar mulheres e recém-nascidos que foram submetidos ao processo de reprodução assistids. Método: Estudo descritivo, retrospectivo para coleta de dados e prospectivo para análise, em mulheres submetidas a duas diferentes técnicas de reprodução assistida no Centro de Reprodução Humana do Hospital Israelita Albert Einstein no período de janeiro de 1995 a Dezembro de 2003. Foram selecionados todos os casos de sucesso e término do parto que ocorreram na Instituição no período. Resultados: no período analisado, ocorreram 2448 procedimentos, sendo 439 de fertilização in vitro e 2009 de injeção intracitoplasmática de esperma. A taxa de sucesso variou de 25 a 30\%. Não houve diferenças significativas entre os dois métodos estudados quanto aos resultados perinatais. Maior morbidade foi observada entre os nascimentos múltiplos em relação aos únicos. Conclusões: as técnicas de reprodução assistida não interferiram nos resultados perinatais. A prematuridade foi o fator determinante de maior morbimortalidade entre os múltiplos.

Palavras-chave: Infertilidade; técnicas reprodutivas; fertilização in vitro; gêmeos; idade gestacional; neonatologia.

\footnotetext{
* $\quad$ Hospital Israelita Albert Einstein - Avenida Albert Einstein, 627 - Morumbi - São Paulo - SP

** Instituto Israelita de Ensino e Pesquisa do Hospital Albert Einstein - Avenida Albert Einstein, 627, Morumbi - São Paulo - SP- conceição@einstein.br

***_Rede Latino Americana de Reprodução Assistida - jonathass.borges@gmail.com

**** Universidade de Campinas - UNICAMP - Departamento de Urologia - sglina@terra.com.br

***** Faculdade de Saúde Pública da Universidade de São Paulo - Departamento Saúde Materno-Infantil - leone.claudio@usp.br

******* Faculdade de Saúde Pública da Universidade de São Paulo - Departamento Saúde Materno-Infantil -Arnaldo Siqueira afsiqueira@uol.com.br

Correspondência para: Marcia de Freitas. Av. Paulista, 66 Apto 52 - Bela Vista - São Paulo, CEP 01310-000. E-mail: mardemar@uol.com.br / marcia.freitas@saude.gov.br
} 


\begin{abstract}
:
Objective: To compare maternal characteristics and perinatal results of children conceived by different techniques of assisted reproduction at the Center of Human Reproduction of Hospital Israelita Albert Einstein. Methods: descriptive study retrospective for data, prospective for analysis in women submitted to different techniques of assisted reproduction at the Center of Human Reproduction of Hospital Israelita Albert Einstein from January 1995 to December 2003. All the cases of pregnancy success and delivery that occurred at the Institution were selected. Results: in the studied period from 2448 procedures were carried out, 439 in vitro fertilization and 2009 intracytoplasmic sperm injection. The pregnancy rate, on average, varied from $25-30 \%$. There was no significant difference between the two methods as to perinatal results. Major morbimortality was observed in multiples in relation to single births. Conclusions: assisted reproduction techniques did not interfere in perinatal results. Prematurity was the determinative factor to the bigger morbimortality of multiple births.
\end{abstract}

Key words: Infertility; reproductive techniques; fertilization in vitro; twins; gestational age; neonatology.

\section{INTRODUÇÃO}

A esterilidade e a infertilidade são doenças devidamente registradas na Classificação Internacional de Doenças - CID 10 (pela OMS) ${ }^{1}$ sendo, portanto, passíveis de tratamento. ${ }^{2}$

Por reprodução humana assistida (RHA) entende-se qualquer ajuda oferecida a um casal com o objetivo de possibilitar que satisfaçam o desejo de alcançar a maternidade ou a paternidade. Essa intervenção pode ir desde o simples aconselhamento sobre o momento mais apropriado do ciclo menstrual para o casal manter relações sexuais, até a utilização de técnicas laboratoriais altamente sofisticadas que permitam a fertilização de um óvulo por um único espermatozóide.

As indicações dessas técnicas estão diretamente ligadas às causas de infertilidade e podem ser denominadas técnicas de baixa complexidade (relação programada e inseminação artificial intra-uterina) ou técnicas de alta complexidade, cujos procedimentos de reprodução assistida envolvem a fertilização extracorpórea (fertilização in vitro clássica-FIVc). Pode ser a fertilização in vitro clássica (FIVc) ou a fertilização in vitro com injeção intracito-plasmática de espermatozóides (ICSI). Ainda como uma "ferramenta coadjuvante" no tratamento de infertilidade temse a criopreservação de espermatozóide e embriões.

Aspecto importante a ser analisado diz respeito ao nascimento de mais uma criança, atualmente considerado um problema de saúde pública, devido aos riscos inerentes às mães, às crianças e ao alto custo gerado ao sistema público de saúde. A mortalidade perinatal, por exemplo, aumenta quatro vezes para a gestação gemelar e seis vezes para a trigemelar. ${ }^{3}$ Taxas mais elevadas de anomalias congênitas são em sua grande maioria, resultados dos efeitos adversos dos nascimentos múltiplos. ${ }^{4,5}$

Como outras alterações causadas pela RHA citam-se ainda, a maior incidência de casos de câncer: há hipóteses de que a gravação defeituosa (defective imprinting) pode ter um papel no desenvolvimento de alguns cânceres da infância, como o tumor de Wilms, o retinoblastoma, e o rabdomiosarcoma embrionário. ${ }^{6,7}$ Há relatos, também, de um possível risco aumentado do câncer ovariano e de mamas em mulheres que receberam terapia de gonadotrofinas. ${ }^{8,9}$ 
Apesar da modalidade ser relativamente recente, diversas organizações internacionais voltadas para a reprodução assistida $\left(\mathrm{CDC}^{10}\right.$, ESHRE $^{11}$ e Rede Lara ${ }^{12}$ ), têm publicado relatórios e artigos referentes aos resultados perinatais das RHA. Porém, mesmo sabendo-se da relevância do assunto, em especial no Brasil, que se destaca mundialmente pelo número de Centros de Reprodução Humana, há poucos estudos sobre a evolução dessas crianças, fato de extrema importância para a complementação e continuidade da assistência prestada, desde o inicio do tratamento da infertilidade à mãe até o nascimento de seu filho. Justificam-se, portanto, em nosso meio, trabalhos que se proponham a estudar variáveis que possam influenciar em alguns eventos obstétricos e perinatais, com ênfase na avaliação de crianças no período neonatal imediato.

Deste modo, o objetivo é caracterizar mulheres e recém-nascidos que foram submetidos ao processo de reprodução assistida.

\section{MÉTODO}

Foi realizado estudo descritivo em mulheres submetidas às diferentes técnicas de reprodução assistida, bem como seu produto de concepção, no período de janeiro de 1995 a dezembro de 2003, cujo parto foi realizado no Hospital Israelita Albert Einstein (HIAE). A amostra inicial foi constituída de 2448 procedimentos, sendo que destes, ocorreram 238 gestações e 322 produtos da concepção, com partos realizados no HIAE. Nesse período, o Serviço de Reprodução Humana HIAE realizou duas modalidades de técnicas de reprodução assistida: fertilização in vitro (FIV) e injeção intracitoplasmática de espermatozóides (ICSI), sendo 45 casos de FIV e 193 casos de ICSI.

O trabalho foi aprovado pela Comissão de Ética em Pesquisa do HIAE (processo CEP - Einstein n ${ }^{\circ}$ 05/359) e pelo Comitê de Ética em Pesquisa da Faculdade de Saúde Pú- blica da Universidade de São Paulo (protocolo de pesquisa $n^{0} 1506$ ).

Os critérios de inclusão foram mulheres submetidas às técnicas de RHA no HIAE e seus recém-nascidos. Os critérios de exclusão da pesquisa foram RN nascidos fora da Unidade Hospitalar do HIAE. Os dados foram coletados do Banco de dados fornecido pela Unidade de Reprodução Humana - HIAE livro de registro dos partos realizados na Instituição, prontuários maternos e dos RN do HIAE (SAME), prontuários digitalizados do Sistema de Informação do HIAE (Sistema de Informação Medtrack) e banco de dados fornecido pelo Serviço de Epidemiologia HIAE.

As variáveis estudadas foram: aquelas referentes às variáveis maternas (idade, doenças maternas, idade gestacional, causas de infertilidade, taxa de sucesso, paridade, taxa de múltiplos, tipo de parto) e a evolução dos seus recém-nascidos, desde o nascimento até a alta do berçário, analisando peso, idade gestacional, boletim Apgar, tempo de internação, malformações encontradas durante a internação e mortalidade neonatal.

As informações coletadas foram colocadas em um banco de dados em ACCESS, sendo exportados os relatórios em EXCEL. A comparação entre duas variáveis categóricas, do tipo grupos da amostra e incidência de determinada característica, foi feita pelo teste de qui-quadrado. Quando a variável em estudo era quantitativa como, por exemplo, o peso do RN, tempo de internação (em dias), etc. utilizou-se o teste de Mann-Whitney para a comparação entre dois grupos e o Kruskal-Wallis para a comparação entre três ou mais grupos. O nível de significância adotado foi de $5 \%$ e todos os testes foram realizados pelo programa estatístico SPSS V11.5.

\section{RESULTADOS}

O Serviço de Reprodução Humana HIAE, no período de janeiro de 1995 a dezem- 
bro de 2003, utilizando duas modalidades de técnicas de reprodução assistida, fertilização in vitro (FIV) e injeção intracitoplasmática de espermatozóides (ICSI), realizou 2.448 procedimentos tendo ocorrido 238 gestações, sendo 45 casos de FIV e 193 casos de ICSI, que resultaram em 322 recém-nascidos vivos.

A tabela 1 demonstra que a população incluída neste estudo foi composta de gestantes com média de idade de 34 anos para FIV e 33,89 anos para ICSI. As médias de idades gestacionais superaram 37 semanas em ambas as técnicas. Estatisticamente, a média da idade materna e da idade gestacional não diferiram significativamente nas duas técnicas es- tudadas, quando analisadas pelo teste de Mann-Whitney.

Afecções maternas durante a gestação ocorreram em $17,6 \%$ das gestantes. Não houve diferença estatística entre as técnicas de RHA em relação à presença de afecções maternas.

Doenças prévias à gravidez ocorreram em $9,7 \%$ das gestantes e não foram estatisticamente significativas quando comparadas por técnicas de RHA $\left(\chi^{2}=1,73 ; \mathrm{P}=0,188\right)$. Nessas pacientes o hipotiroidismo foi a doença com maior freqüência $(3,4 \%)$, seguido de abortamentos de repetição (0,8\%). Durante o procedimento de RHA encontrou-se um caso de síndrome de hiperestimulação ovariana.

Tabela 1: Distribuição das gestantes segundo idade materna, idade gestacional e técnica de Reprodução Humana Assistida

\begin{tabular}{lcrrrrrr}
\hline \multicolumn{1}{c}{ Idade } & Técnica & N & Mediana & Média & $\begin{array}{c}\text { Desvio } \\
\text { padrão }\end{array}$ & $\begin{array}{c}\text { Mann- } \\
\text { Whitney } \\
\text { (Z) }\end{array}$ & $\begin{array}{c}\text { Probabi- } \\
\text { lidade }\end{array}$ \\
\hline Idade Materna & FIVc & 45 & 34 & 34,00 & 4,47 & $-0,073$ & 0,942 \\
& ICSI & 193 & 34 & 33,89 & 4,06 & & 0,473 \\
Idade Gestacional & FIVc & 45 & 38 & 37,7 & 1,95 & $-0,717$ & 0,473 \\
& ICSI & 193 & 37,5 & 37,41 & 2,59 & & \\
\hline
\end{tabular}

Fonte: Unidade de Reprodução Assistida - Hospital Israelita Albert Einstein.

Afecções maternas durante a gravidez ocorreram em $17,6 \%$ das pacientes da amostra, não se observando diferenças estatisticamente significativas entre as técnicas.

As doenças maternas geraram $31,1 \%$ de internações no HIAE. A causa mais freqüente de internação $(11,3 \%)$ foi o trabalho de parto prematuro (TPP), seguido de amniorrexe prematura (6,7\%). A afecção incompetência istmo-cervical correspondeu a 2,5\% das causas de internação para realização de circlagem uterina. No entanto $100 \%$ dessas pacientes apresentaram trabalho de parto prematuro. Hipertensão arterial especifica da gravidez ocorreu em 5,9\% das gestantes e diabetes gestacional em 4,2\%.

Em relação à paridade, 59,2\% das gestantes eram primigestas. Abortos ocorreram em mais de $20 \%$ na população geral do estudo. No entanto a paridade e o número de abortos não foram estatisticamente diferentes para as duas técnicas de RHA.

As taxas de sucesso são apresentadas na tabela 2, subdivididas em taxa de gravidez bioquímica, clínica e efetiva, analisadas segundo as técnicas.

A tabela 2 demonstra que não houve diferença significativa entre as técnicas. Apesar do resultado estatístico encontrado na amostra, ressalta-se um decréscimo de cada fase de evolução de gravidez. O que chama a atenção é um decréscimo de aproximadamente $30 \%$ da gravidez bioquímica para a gravidez efetiva.

Quanto ao tipo de parto, houve predominância do parto cesárea nas gestantes dos dois grupos, que correspondeu a 92,0\% de toda a amostra. O tipo de parto não diferiu estatisticamente nos dois tipos de técnicas de RHA conforme mostrado pelo teste de qui-quadrado. $\left(\chi^{2}=1,651 ; \mathrm{P}=0,438\right)$. 
Tabela 2: Taxas de sucesso segundo os procedimentos realizados

\begin{tabular}{lrcrccc}
\hline $\begin{array}{l}\text { Taxa de Gravidez } \\
\text { (PE transferidos) }\end{array}$ & \multicolumn{2}{c}{$\begin{array}{c}\text { FIV } \\
(\mathbf{n}=\mathbf{3 5 9})\end{array}$} & \multicolumn{2}{c}{$\begin{array}{c}\text { ICSI } \\
(\mathbf{n}=\mathbf{1 7 1 2})\end{array}$} & $\boldsymbol{\chi}^{\mathbf{2}}$ & $\mathbf{P}$ \\
\hline & $\mathbf{n}$ & $\mathbf{\%}$ & \multicolumn{2}{c}{$\mathbf{\%}$} & & \\
\hline Gravidez bioquímica & 116 & 32,3 & 482 & 28,2 & 1,213 & 0,271 \\
Gravidez clínica & 104 & 29,0 & 413 & 24,1 & 1,994 & 0,158 \\
Gravidez efetiva & 81 & 22,6 & 303 & 17,7 & 2,861 & 0,091 \\
\hline
\end{tabular}

Fonte: Unidade de Reprodução Assistida - Hospital Israelita Albert Einstein.

Na tabela 3 encontra-se a distribuição do número de RN por gestação e demonstra a ocorrência do percentual de gestações múltiplas (31,1\%), não havendo diferenças estatisticamente significativas entre as técnicas.
Quanto às características do RN, na população do estudo, segundo a razão masculino/feminino para cada técnica, foi de 1.09 para os RN de FIV e 0, 97 para os RN de ICSI, sem diferença significativa entre as técnicas $\left(\chi^{2}=\right.$ $0,170 ; \mathrm{P}=0,680)$.

Tabela 3: Distribuição do número de recém-nascido por gestação

\begin{tabular}{lrrrrrr}
\hline \multirow{2}{*}{ Tipo de gestação } & \multicolumn{2}{c}{ FIVc } & \multicolumn{2}{c}{ ICSI } & \multicolumn{2}{c}{ Total } \\
\hline & $\mathbf{N}$ & $\mathbf{\%}$ & $\mathbf{n}$ & $\mathbf{\%}$ & $\mathbf{n}$ & $\mathbf{\%}$ \\
\hline Única & 27 & 60,0 & 137 & 71,0 & 164 & 68,9 \\
Duplo & 15 & 33,3 & 51 & 26,4 & 66 & 27,7 \\
Trigemelar $(* 1$ quadrigême0) & 3 & 6,7 & $5 *$ & 2,6 & 8 & 3,4 \\
Total & 45 & 100 & 188 & 100 & 238 & 100 \\
\hline
\end{tabular}

$\chi^{2}=3,071 ; \mathrm{P}=0,215$

A classificação do RN segundo o crescimento intra-uterino, evidenciou que $7,1 \%$ dos RN estavam acima do percentil 90 da curva de crescimento grande para idade gestacional (GIG) e $5 \%$ ficou abaixo do percentil 10, pequeno para idade gestacional (PIG). Os RN apropriados para a idade gestacional (AIG) corresponderam à maioria nos grupos analisados.

Encontraram-se dois RN de tamanho excessivamente grande (peso > 4500g), ambos filhos de gestantes diabéticas.

Verificou-se que 33,9\% ( $n=109) \mathrm{RN}$ nasceram com peso menor de $2500 \mathrm{~g}$, sendo que $13,7 \%(n=15)$ destes RN tiveram seu peso menor de 1500g. Observou-se também que, em relação ao peso do nascimento, não se encontrou diferença significativa entre as duas técnicas de RHA $\left(\chi^{2}=5,501 ; \mathrm{p}=0,139\right)$.
Observa-se na tabela 4 a distribuição do número dos RN por gestação. Segundo a idade gestacional, a prematuridade (idade gestacional $<37$ semanas) foi aumentando à medida que se elevou o número de conceptos nascidos por gestação.

A tabela 5 demonstra características selecionadas dos $\mathrm{RN}$, com as variáveis peso (nascimento e alta), boletim Apgar (1 e 5 minutos) e número de dias de internação quando comparadas pelo teste de Mann-Whitney para ambas as técnicas, que nenhuma dessas variáveis apresentou diferença significativa entre as duas técnicas.

Malformações congênitas foram evidenciadas em $10,6 \%$ dos RN, mais freqüentes com o avanço da idade materna $\left(\chi^{2}=1,793\right.$; $\mathrm{p}=0,617)$, porém não se evidenciaram dife- 
Tabela 4: Distribuição do número dos recém-nascidos por gestação segundo a idade gestacional

\begin{tabular}{lcccccccc}
\hline Idade Gestacional & \multicolumn{2}{c}{ Único } & \multicolumn{2}{c}{ Duplo } & \multicolumn{2}{c}{ Trigêmeo* } & \multicolumn{2}{c}{ Total } \\
\hline & $\mathrm{n}$ & $\%$ & $\mathrm{n}$ & $\%$ & $\mathrm{n}$ & $\%$ & $\mathrm{n}$ & $\%$ \\
\hline \multirow{2}{*}{28} & 1 & 0,6 & 4 & 3,0 & 3 & 12,0 & 8 & 2,5 \\
$29-36$ & 149 & 90,3 & 54 & 40,9 & 5 & 20,0 & 208 & 64,6 \\
$\geqslant 37$ & 15 & 9,1 & 74 & 56,1 & 17 & 68,0 & 106 & 32,9 \\
Total & 165 & 100,0 & 132 & 100,0 & 25 & 100,0 & 322 & 100,0 \\
\hline
\end{tabular}

$\chi^{2}=95,561 ; \mathrm{P}<0,001$

*acrescidos os quadrigêmeos

renças estatisticamente significativas entre as técnicas de RHA. (FIV $\chi^{2}=2,400 ; \mathrm{p}=0,494$ e ICSI $\left.\chi^{2}=2,293 ; \mathrm{p}=0,514\right)$.

As malformações cardíacas foram às alterações mais freqüentemente encontradas. Chamou atenção o encontro de malformações maiores, como no caso da seqüência de VACTERL (2 casos) e cardiopatias congênitas complexas (6 casos). Não houve nenhum óbito entre os únicos e entre os múltiplos, foram 12 (5,0\%), sendo 8 duplos (8/66) e 4 trigêmeos (4/8).

Tabela 5: Características dos Recém-Nascidos segundo variáveis peso, apgar e tempo de internação

\begin{tabular}{|c|c|c|c|c|c|c|c|}
\hline Variável & RHA & $\mathbf{N}$ & Mediana & Média & $\begin{array}{l}\text { Desvio } \\
\text { padrão }\end{array}$ & $\begin{array}{c}\text { Mann- } \\
\text { Whitney } \\
\text { (Z) }\end{array}$ & $\mathbf{P}$ \\
\hline \multirow[t]{2}{*}{ Peso do RN no nascimento } & ICSI & 255 & 2740 & 2704,1 & 745,4 & $-0,136$ & 0,892 \\
\hline & FIVc & 67 & 2680 & 2726,3 & 622,2 & & \\
\hline \multirow[t]{2}{*}{ Peso na alta } & ICSI & 240 & 2535 & 2596,6 & 733,11 & $-0,194$ & 0,847 \\
\hline & FIVc & 61 & 2540 & 2614,3 & 505,3 & & \\
\hline \multirow[t]{2}{*}{ Apgar 1} & ICSI & 248 & 9 & 8,23 & 1,556 & $-0,523$ & 0,601 \\
\hline & FIVc & 64 & 9 & 9,45 & 10,33 & & \\
\hline \multirow[t]{2}{*}{ Apgar 5} & ICSI & 248 & 10 & 9,31 & 1,42 & $-0,909$ & 0,363 \\
\hline & FIVc & 64 & 10 & 9,44 & 1,32 & & \\
\hline \multirow[t]{2}{*}{ Dias de internação } & ICSI & 255 & 3 & 7,85 & 15,92 & $-1,522$ & 0,128 \\
\hline & FIVc & 67 & 4 & 11,72 & 45,52 & & \\
\hline
\end{tabular}

\section{DISCUSSÃO}

A infertilidade de causa feminina tem sido muito mais estudada na literatura em relação à masculina. ${ }^{13}$ Observou-se no presente estudo, que analisou tanto causas femininas como masculinas, que houve predomínio das causas ligadas à infertilidade feminina. $\mathrm{O}$ fator tubo-peritonial, a iatrogenia e o fator ovulatório colocam-se como os mais freqüen- tes fatores de infertilidade. ${ }^{2}$ No presente trabalho também foi constatado predomino do fator tubário. Em 2006, segundo dados do $\mathrm{CDC}^{10}$, o fator feminino de um modo geral (incluindo o tubário) foi responsável por 32,9\% dos casos, o fator masculino foi responsável por 18,8\% e a infertilidade mista por $17,9 \%$ dos casos. No presente trabalho os dados se mostraram semelhantes aos do CDC. ${ }^{10}$ 
Entre os fatores de infertilidade masculina na população em estudo, a grande maioria se deveu à azoospermia, conforme referido na literatura. ${ }^{10}$

Quanto à idade materna, sabidamente fator preponderante para infertilidade, constatou-se pelo presente estudo a existência de um elevado percentual de mulheres com idade superior a 35 anos, o mesmo se verificando com a idade dos homens. A infertilidade nessas mulheres pode ocorrer principalmente por perdas de fetos com trissomias e euploidias, resultado de um declínio na qualidade do oócito. ${ }^{10}$

Quanto às repercussões da idade paterna avançada sobre o concepto ainda há controversias. ${ }^{14}$ Alguns estudos mostram que a idade paterna está associada ao aumento de mutações autossômicas dominantes (por exemplo, acondroplasia, síndromes de Apert e Marfan) que poderiam resultar em anomalias congênitas para os seus descendentes. ${ }^{15}$

Das doenças que ocorrem na gestação deve-se assinalar que a RHA é por si só importante causa de morbidade materna. ${ }^{16}$ Entre essas: sangramento vaginal após 28 semanas, placenta prévia; oligoidrâmnio; insuficiência placentária e a síndrome de hiperestimulação do ovário. Esta última afecção também foi encontrada no presente trabalho. A explicação para esse número aumentado de complicações poderia estar ligada às alterações endometriais, sabidamente presentes nessas mulheres. ${ }^{16}$

Shevell et al. ${ }^{16}$ analisaram 36.062 mulheres e constataram que a indução da ovulação estava associada estatisticamente com o aumento de descolamento prematuro de placenta, perda fetal após 24 semanas e diabetes gestacional, enquanto que nas pacientes que realizaram FIVc, houve uma associação estatisticamente significativa com o aumento de pré-eclâmpsia, hipertensão gestacional, descolamento prematuro de placenta, placenta prévia e maior risco de parto cesárea.
No presente trabalho, não foram encontradas diferenças estatisticamente significativas em relação a doenças maternas, independentemente das técnicas utilizadas.

Hipertensão e pré-eclâmpsia ocorrem muito freqüentemente na gestação múltipla, podendo haver um risco 2,1 maior em relação às gestações espontâneas. ${ }^{17}$ Burkmaan et $\mathrm{al}^{9}$ constataram $24 \%$ de eclâmpsia grave, em gestações triplas com 20 semanas de idade gestacional, incluindo a forma mais grave dessa doença onde ocorre hemólise e com testes de função hepática alteradas. Ainda podese incluir entre as complicações maternas o polidrâmnio, que pode ocorrer em $12 \%$ das gestações múltiplas, além de aumentar o risco de parto prematuro. Outras afecções que também estão associadas à gestação múltipla são a hiperêmese gravídica, e o refluxo gastroesofágico. Resultados bastante adversos, também foram encontrados em uma revisão bibliográfica para avaliação do impacto das técnicas de fertilização assistida na morbidade materna e perinatal. ${ }^{18}$

As complicações maternas, que ocorreram em $17,6 \%$ das gestantes, apresentaram freqüência menor que as relatadas na literatura, podendo-se especular que esse fato esteja ligado à boa qualidade do pré natal.

A anemia, que pode ocorrer agudamente na gestação ou até mesmo no puerpério ${ }^{18}$, no presente trabalho, não representou agravo importante, sendo que o trabalho de parto prematuro foi a principal causa de internação durante a gestação.

Em relação ao número de abortos, não houve diferenças significativas entre as técnicas estudadas.

Schieve e Reynolds ${ }^{19}$ relatam que a taxa de sucesso, ou seja, de ciclos iniciados que resultaram em gravidez, com $\mathrm{RN}$, foi de 34\% com a transferência de dois embriões. Em publicação de Rede LARA ${ }^{12}$ a taxa de parto por transferência de embriões frescos para fertilização in vitro (FIV), injeção 
intracitoplasmática de espermatozóides (ICSI) e FIV+ICSI foi de $24,5 \%$.

As taxas de sucesso efetivo aqui encontradas foram inferiores às relatadas, contudo alguns fatores concomitantes podem explicar esse achado, como a ocorrência de grande número de mulheres com idade avançada, a paridade e a transferência média de menos de três embriões por procedimento.

No presente estudo observou-se uma elevada frequência de parto cesárea nas mulheres submetidas à RHA, embora sem diferenças significativas entre as técnicas estudadas. Prende-se o fato a vários fatores, desde aqueles relatados de risco materno, até a decisões do próprio casal. ${ }^{18}$

Observou-se que embora a proporção de gestações dupla e trigemelar, estivessem acima do encontrado em pacientes não submetidas à RHA, a ocorrência de gestação múltipla não mostrou diferenças significativas entre as duas técnicas.

Em relação aos aspectos neonatais, não houve diferenças significativas quanto ao sexo dos RN em relação às técnicas de RHA, o que está de acordo com a literatura. ${ }^{20}$

Quanto à população geral, a literatura relata um aumento da incidência de prematuridade, não somente entre os múltiplos, como também entre os RN de parto único concebidos de técnicas de RHA. ${ }^{21}$

Apesar do critério "termo" não pressupor a maturidade plena do concepto, a maioria das crianças neste trabalho foi classificada como RN de termo (37semanas ou mais de idade gestacional), sem diferenças significativas entre os dois procedimentos analisados. Todavia, comparando-se com dados brasileiros, deve-se salientar que a taxa de prematuridade no Brasil, segundo o IBGE ${ }^{22}$, em 2006, foi aproximadamente cinco vezes menor do que a taxa aqui encontrada.

O número de crianças com restrição de crescimento intra-uterino não mostrou diferença entre as duas técnicas estudadas. Buckett et al. ${ }^{23}$ também não referem haver diferença significativa entre as técnicas de RHA e a ocorrência de restrição de crescimento intrauterino.

Em relação aos RN, em geral, parece não haver associação com complicações graves, em especial as relacionadas ao desenvolvimento neuro-psico-motor no $\mathrm{RN}$ de termo que nasceu de algumas das técnicas de RHA em relação aos RN concebidos naturalmente. No entanto, o mesmo não ocorre nas gestações múltiplas, onde se encontram os dois maiores fatores de risco para ocorrência de seqüelas neurológicas, a prematuridade e o baixo peso de nascimento. Dentre as afecções neurológicas no RN, ganham destaque a paralisia cerebral, hemorragia intraventricular e as alterações motoras e cognitivas. Além de todas as complicações já mencionadas, foi observada maior incidência de algumas neoplasias. Encontra-se também uma elevada incidência de malformações maiores. ${ }^{20}$

Entretanto, existem estudos recentes que contestam essas afirmativas, pois encontraram os mesmos riscos que na população geral, principalmente no que se refere ao desenvolvimento psico-social. ${ }^{24}$

Não foram encontradas diferenças significativas em relação ao boletim Apgar de $1^{\circ}$ e $5^{\circ}$. minutos entre os RN, quanto ao tempo de permanência e nem em relação ao peso de alta, podendo-se concluir que a evolução na unidade neonatal tenha sido semelhante entre os RN analisados segundo as técnicas estudadas.

O encontro de malformações não mostrou diferenças significativas entre únicos e gemelares, embora represente número elevado em relação ao relatado para uma população geral e é achado compatível com a literatura. ${ }^{23}$ No presente trabalho encontrou-se um maior número de pé torto congênito, também demonstrado em um estudo realizado em 2500 RN que analisou malformações congênitas das extremidades em três maternidades da cidade de Maceió. Esse estudo mostrou que a anomalia mais freqüente foi o pé torto eqüinovaro com uma prevalência 
de 7,2 para 1000 nascidos vivos, sendo mais freqüente no sexo masculino. ${ }^{25}$

Em relação à mortalidade neonatal, foram encontradas, na amostra, diferenças importantes entre os gêmeos e trigêmeos quando comparados aos únicos. Não houve óbitos entre os RN de gestação única de RHA. Corroborando este achado, Kahn et al. ${ }^{26}$, em estudo prospectivo, analisaram 11.061.599 de gestações únicas, 297.622 gemelares e 15.375 triplas, a partir dos registros do National Center for Health Statistics americano entre 1995 e 1998, concluindo, entre outros aspectos, que o risco de morte fetal para gêmeos e triplos era significativamente maior que para únicos. Assim, podese inferir que a elevada mortalidade neonatal, segundo o que já foi exposto em relação aos riscos da gemelaridade ${ }^{3}$, está mais ligada às complicações dos nascimentos múltiplos que às técnicas de RHA propriamente ditas.

As técnicas de RHA têm apresentado ao longo dos anos grandes avanços. Contudo, em nosso meio, o acompanhamento do RN de RHA ainda se ressente de maior aprofundamento. Não se discute que a necessidade da elaboração de

\section{REFERÊNCIAS}

1. OMS - Organização Mundial da Saúde. Manual da classificação estatística internacional de doenças e problemas relacionados à saúde: $10^{a}$ revisão. São Paulo:Edusp; 1997.

2. Glina, S, Soares JB, Meirelles AJC, Antunes Jr A. Reprodução humana. In Segre CAM. Perinatologia.

Fundamentos e prática.-São Paulo: Sarvier; 2002.p. 3-14.

3. Gunby J, Daya S. IVF Directors Group of the Canadian Fertility and Andrology Society. Assisted reproductive technologies (ART) in Canada: 2001 results from the Canadian ART Register. Fertil Steril. 2005. 84(3):590-9. trabalhos que levem em conta o seguimento especializado dessas crianças nos primeiros anos de vida é mandatória. Mas, indo além, muitos estudos ainda são necessários focalizando as habilidades cognitivas, desempenho escolar e desenvolvimento emocional de crianças e adolescentes de RHA. O estudo do RN de RHAé apenas o começo do caminho por uma longa estrada.

Assim, as mulheres submetidas à RHA no HIAE apresentaram, em média, idade avançada e a principal causa de infertilidade foi a feminina, relacionada ao fator tubário. A injeção intracitoplasmática de espermatozóides constitui-se na técnica mais freqüente. Os RN não diferiram, segundo as técnicas estudadas, quanto ao peso de nascimento, idade gestacional, vitalidade ao nascer, bem como, classificação do peso pela idade gestacional, peso de alta e tempo de permanência hospitalar. Encontrou-se elevada proporção de malformações entre os RN dessa população, mas não diferiram significativamente em relação às técnicas empregadas. A mortalidade neonatal mostrou-se elevada em relação aos múltiplos quando comparados aos únicos.

4. Reddy UM, Wapner RJ, Rebar RW, Tasca RJ. Infertility, assisted reproductive technology, and adverse pregnancy outcomes: executive summary of a National Institute of Child Health an Human Development workshop. Obstet Gynecol. 2007; 109(4): 967-77.

5. Jones KL. Smith's recognizable patterns of human malformation. 6th ed.

Philadelphia: Elsevier Saunders; 2006.

6. Klip H, Burger CW, de Kraker J, van Leeuwen FE. Risk of cancer in the offspring of women who underwent ovarian stimulation for IVF. Hum Reprod. 2001;16(11):2451-8. 
7. Lerner-Geva L, Toren A, Chetrit A, Modan B, Mandel M, Rechav G, Dor J. The risk for cancer among children of women who underwent in vitro fertilization. Cancer. 2000; 88(12):2845-7.

8. Brinton LA, Lamb EJ, Moghissi KS, Scoccia B, Althuis M, Mabie J, Westhoff C. Ovarian cancer risk associated with varying causes of infertility. Fertil Steril 2004; 82(2):405-14.

9. Burkman RT, Tang MC, Malone KE, Marchbanks PA, McDonald JA, Folger SG, et al. Infertility drugs and the risk of breast cancer: findings from the National Institute of Child Health and Human Development Women's Contraceptive and Reproductive Experiences Study. Fertil Steril. 2003; 79(4):844-51.

10. CDC- Centers for Disease Control. Assisted Reproductive Technology. 2007. [texto na Internet] 2006 [citado 2008 jan10]. Disponível em: <http:// www.cdc.gov/art/>

11. ESHRE - European Society of Human Reproduction \& Embriology [texto na Internet] 2006 [citado 2008 jan10]. Disponível em: http://www.eshre.com/ emc.asp?pageId=195

12. Rede Lara - Rede Latino-Americana de Reprodução Assistida. Registro Latinoamericano da. [texto na Internet] 2006 [citado 2008 jan10]. Disponível em: http://www.redlara.com/ REGISTRO.ASP

13. Olmed B, Chllik C, Kopelman S. Definición y causas de la infertilidad. Rev Colomb Obstet Ginecol. 2003; 54(4):1-3.

14. Kazaura M, Lie RT, Skjaerven R. Paternal age and the risk of birth defects in Norway. Ann Epidemiol. 2004; 14(8):566-60.
15. Rolf C, Nieschlag E. Reproductive functions, fertility and genetic risks of ageing men. Exp Clin Endocrinol Diabetes. 2001;109(2):68-74.

16. Shevell T, Malone FD, Vidaver J, Porter TF, Luthy DA, Comstroch CH et al. Assisted reproductive technology and pregnancy outcome. Obstet Gynecol. 2005;106(5 Pt 1):1039-45.

17. Lynch A., McDuffie R, Jr, Murphy J, Faber K, Orleans M. Preeclampsia in multiple gestation: The role of assisted reproductive technologies. Obstet Gynecol. 2002;99(3):445-51.

18. Silva JLP, Bahamondes L. Reprodução assistida como causa de morbidade materna e perinatal. Rev Bras Ginecol Obstet. 2005. 27(12): 759-67.

19. Schieve LA, Reynolds MA What is the most relevant standard of success in assisted reproduction? Challenges in measuring and reporting success rates for assisted reproductive technology treatments: what is optimal? Hum Reprod. 2004;19(4):778-82.

20. Bonduelle M, Ponjaert I, Steirteghem AV, Derde MP, Devroey P, Liebaers I. Developmental outcome at 2 years of age for children born after ICSI compared with children born after IVF. Hum Reprod. 2003;18(2):342-50.

21. Schieve LA, Ferre C, Peterson HB, Macaluso M, Reynolds MA, Wright VC. Perinatal outcome among singletons conceived through assisted reproductive technology in the United States. Obstet Gynecol. 2004;103(6):1144-53. 
22. IBGE Instituto Brasileiro de Geografia e Estatística. Sidra - Tabela 2466 - pessoas de 10 anos ou mais por estado civil, situação de domicilio, condição de convivência e grupos. [texto na Internet] 2006 [citado 2008 jan10]. Disponível em $<$ http://www.sidra.ibge.gov.br/bda/ tabela/listabl.asp?z=t\&c=2466>

23. Buckett WM, Chian RC, Holzer H, Dean N, Usher R, Tan SL. Obstetric outcomes and congenital abnormalities after in vitro maturation, in vitro fertilization, and intracytoplasmic sperm injection. Obstet Gynecol. 2007;110(4):885-91.

24. Wagenaar K, Huisman J, Cohen-Kettenis PT, Delemarre-van de Waal HA. An overview of studies on early development, cognition, and psychosocial well-being in children born after in vitro fertilization. J Dev Behav Pediatr. 2008;29(3):219-30. Review.
25. Pereira RJdaS, Abreu Lcde, Valenti VE, Albuquerque WDM, Pereira SC, Araújo R, Ferreira NetoML, Santos SMdos. Freqüência de malformações congênitas das extremidades em recém-nascidos. Rev Bras Crescimento Desenvol Hum. 2008; 18(2): 155-162.

26. Kahn B, Lumey LH, Zybert PA, Lorenz JM, Cleary-Goldman J, D’Alton ME, et al. Prospective risk of fetal death in singleton, twin, and triplet gestations: implications for practice. Obstet Gynecol. 2003;102(4):685-92.
Recebido em: 20/08/2008 Modificado em: 18/11/2008 Aceito em: 12/12/2008 